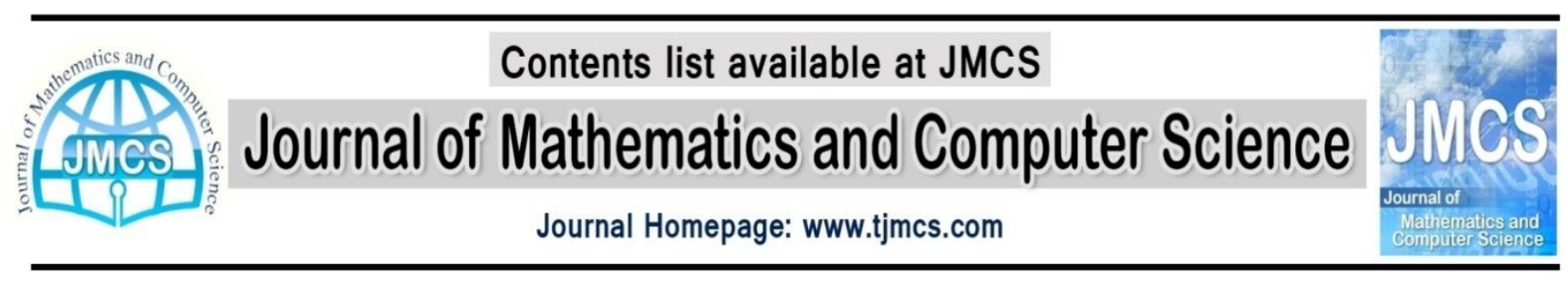

\title{
Fuzzy Multi-objective Linear Programming Problems: Sensitivity Analyses
}

\author{
M. Pattnaik \\ Dept. of Business Administration, \\ Utkal University, Bhubaneswar-751004, India, \\ monalisha_1977@yahoo.com
}

Article history:

Received March 2013

Accepted Apri 2013

Available online April 2013

\begin{abstract}
Thanks to global competition, faster product development, and increasingly flexible manufacturing systems, an unprecedented number and variety of products are competing in markets ranging from apparel and toys to power tools and computers. The dramatic increase in demand unpredictability is fairly recent, in practice, there are many problems in which all decision parameters are fuzzy numbers, and such problems are usually solved by either probabilistic programming or multiobjective programming methods. Unfortunately all these methods have shortcomings. In this note, using the concept of comparison of fuzzy numbers, a very effective method is introduced for solving these problems. This paper extends linear programming based problem in fuzzy environment. With the problem assumptions, the optimal solution can still be theoretically solved using simplex based method. To handle the fuzzy decision variables can be initially generated and then solved and improved sequentially using the fuzzy decision approach by introducing Robust's ranking technique. The proposed procedure was programmed and the three dimensional mesh plot diagram is represented through MATLAB (R2009a) version software. The model is illustrated with numerical example and a sensitivity analyses are of the optimal solution is studied with respect to changes in parameter which incorporates all concepts of a fuzzy arithmetic approach to draw managerial insights.
\end{abstract}

Keywords: Multi-objective, Linear programming, Fuzzy number, Simplex method, Sensitivity analysis.

\section{Introduction}

With many different perspectives around the world, the "right" choice is not clear, so the manager makes decisions in large part by understanding the concerns of its real life problems. With many different Linear programming is the optimization technique most frequently applied in real-world problems and there it is important to introduce new tools in the approach that allow the model to fit into the real world as much as possible. Any linear programming model representing real-world situations involves a lot of parameters whose values are assigned by experts, and in the conventional approach, they are required to fix an exact value to the aforementioned parameters. However, both experts and the decision maker frequently do not precisely know the value of those parameters. If exact values are suggested these are only statistical inference from past data and their stability is doubtful, so the parameters of the problem are usually defined by the decision maker in an uncertain space. Therefore, it is useful to consider the knowledge of experts about the parameters as fuzzy. Two significant questions may be found in these 
kinds of problems: how to handle the relationship between the fuzzy parameters, and how to find the optimal values for the fuzzy multi-objective function. The answer is related to the problem of ranking fuzzy numbers.

In fuzzy decision making problems, the concept of maximizing decision was introduced by [2]. [17] presented a fuzzy approach to multi-objective linear programming problems in his classical paper. [6] considered the situations where all parameters are in fuzzy. [5] assume that the parameters have a triangular possibility distribution. [3] introduce fuzzy linear programming problem based on L-R fuzzy number. [4] propose a method for solving linear programming problems where all coefficients are, in general, fuzzy numbers and using linear ranking technique. [1], [9], [10] and [16] define linear programming problems with fuzzy numbers and simplex method is used for finding the optimal solution of the fuzzy problem. [12] compute improved fuzzy optimal Hungarian assignment problems with fuzzy numbers by applying Robust's ranking techniques to transform the fuzzy assignment problem to a crisp one. [11] presented a fuzzy approach to several linear and nonlinear inventory models. [14] explain the method to obtain sensitivity analysis or post optimality analysis of the different parameters in the linear programming problems.

Looking at the property of representing the preference relationship in fuzzy terms, ranking methods can be classified into two approaches. One of them associates, by means of different functions, each fuzzy number to a single of the real line and then a total crisp order relationship between fuzzy numbers is established. The other approach ranks fuzzy numbers by means of a fuzzy relationship. It allows decision maker to present his preference in a gradual way, which in a linear programming problem allows it to be handled with different degrees of satisfaction of constraints. This paper considers fuzzy multi-objective linear programming problems whose parameters are fuzzy numbers but whose decision variables are crisp. The aim of this paper is to introduce Robust's ranking technique for defuzzifying the fuzzy parameters and then sensitivity analysis for requirement vector in the constraint function is also performed that permits the interactive participation of decision maker in all steps of decision process, expressing his opinions in linguistic terms. The major techniques used in the above research articles are summarized in Table 1.

Table 1 Major Characteristics of Fuzzy Linear Programming Models on Selected Researches

\begin{tabular}{|c|c|c|c|c|c|}
\hline $\begin{array}{c}\text { Author(s) and } \\
\text { Published Year }\end{array}$ & $\begin{array}{c}\text { Structure of } \\
\text { the Model }\end{array}$ & $\begin{array}{c}\text { Objective } \\
\text { Model }\end{array}$ & Model Type & $\begin{array}{c}\text { Ranking } \\
\text { Function }\end{array}$ & $\begin{array}{c}\text { Sensitivity } \\
\text { Study }\end{array}$ \\
\hline $\begin{array}{c}\text { Zimmermann } \\
(1978)[17]\end{array}$ & Fuzzy & Single & Cost & Linear & No \\
\hline $\begin{array}{c}\text { Maleki et al. } \\
(2000)[8]\end{array}$ & Fuzzy & Multi & Profit & Linear & No \\
\hline $\begin{array}{c}\text { Jimenez et al. } \\
(2005)[4]\end{array}$ & Fuzzy & Multi & Cost & Linear & No \\
\hline $\begin{array}{c}\text { Nasseri et al. } \\
(2005)[9]\end{array}$ & Fuzzy & Multi & Profit & Linear & No \\
\hline $\begin{array}{c}\text { Present Paper } \\
(2012)\end{array}$ & Fuzzy & Multi & Profit & Robust & Yes \\
\hline
\end{tabular}

The remainder of this paper is organized as follows. In Section 2, fuzzy numbers and some of the results of applying arithmetic on them are introduced. Assumptions, notations and definitions are provided for the development of the model. In Section 3, Robust's ranking technique is implemented for solving fuzzy number linear programming problems. In Section 4, a linear programming problem is proposed with fuzzy variables and explained a method for solving this problem. The numerical example is presented to illustrate the development of the model in section 5. The sensitivity analyses are carried out in section 6 to observe the changes in the optimal solution. Finally section 7 deals with the summary and the concluding remarks. 


\section{Preliminaries}

The fundamental notation of fuzzy set theory is reviewed and it is initiated by [2]. Below definitions are given which are abstracted from [17].

\section{Definition 2.1 Fuzzy sets}

If $\mathrm{X}$ is a collection of objects denoted generally by $x$, then a fuzzy set $\tilde{A}$ in $\mathrm{X}$ is defined as a set of ordered pairs $\tilde{A}=\left\{\left(x, \mu_{\tilde{A}}(x)\right) / x \in X\right\}$, where $\mu_{\tilde{A}}(x)$ is called the membership function for the fuzzy set $\tilde{A}$. The membership function maps each element of $X$ to a membership value between 0 and 1 .

Definition 2.2 Support of a fuzzy set

The support of a fuzzy set $\tilde{A}$ is the set of all points $x$ in $\mathrm{X}$ such that $\mu_{\tilde{A}}(x)>0$. That is $\operatorname{support}(\tilde{A})=\left\{x / \mu_{\tilde{A}}(x)>0\right\}$.

Definition 2.3 $\alpha$-level of fuzzy set

The $\alpha$ - cut (or) $\alpha$ - level set of a fuzzy set $\tilde{A}$ is a set consisting of those elements of the universe $\mathrm{X}$ whose membership values exceed the threshold level $\alpha$. That is $\tilde{A}_{\alpha}=\left\{x / \mu_{\tilde{A}}(x) \geq \alpha\right\}$.

Definition 2.4 Convex fuzzy set

A fuzzy set $\tilde{A}$ is convex if, $\mu_{\tilde{A}}\left(\lambda x_{1}+\left(1-\lambda x_{2}\right) \geq \min \left(\mu_{\tilde{A}}\left(x_{1}\right), \mu_{\tilde{A}}\left(x_{2}\right)\right), x_{1}, x_{2} \in X\right.$ and $\lambda \epsilon[0,1]$. Alternatively, a fuzzy set is convex, if all $\alpha$-level sets are convex.

Definition 2.5 Convex normalized fuzzy set

A fuzzy number $\tilde{A}$ is a convex normalized fuzzy set on the real line $\mathrm{R}$ such that it exists at least one $x_{0} \in R$ with $\mu_{\tilde{A}}\left(x_{0}\right)=1$ and $\mu_{\tilde{A}}(x)$ is piecewise continuous.

\section{Definition 2.6 Trapezoidal fuzzy numbers}

Among the various fuzzy numbers, triangular and trapezoidal fuzzy numbers are of the most important. Note that, in this study only trapezoidal fuzzy numbers are considered. A fuzzy number is a trapezoidal fuzzy number if the membership function of its be in the following function of it being in the following form:

Any trapezoidal fuzzy number by $\tilde{a}=\left(a^{L}, a^{U}, \alpha, \beta\right)$, where the support of $\tilde{a}$ is $\left(a^{L}-\alpha, a^{U}+\beta\right)$ and the modal set of $\tilde{a}$ is $\left[a^{L}, a^{U}\right]$. Let $F(R)$ is the set of trapezoidal fuzzy numbers.

Definition 2.7 Arithmetic on fuzzy numbers

Let $\widetilde{a}=\left(a^{L}, a^{U}, \alpha, \beta\right)$ and $\tilde{b}=\left(b^{L}, b^{U}, \gamma, \theta\right)$ be two trapezoidal fuzzy numbers and $x \in R$. Then, the results of applying fuzzy arithmetic on the trapezoidal fuzzy numbers as shown in the following:

Image of $\widetilde{a}:-\widetilde{a}=\left(-a^{U},-a^{L}, \beta, \alpha\right)$

Addition: $\tilde{a}+\tilde{b}=\left(a^{L}+b^{L}, a^{U}+b^{U}, \alpha+\gamma, \beta+\theta\right)$

Scalar Multiplication: $x>0, x \widetilde{a}=\left(x a^{L}, x a^{U}, x \alpha, x \beta\right)$ and $x<0, x \widetilde{a}=\left(x a^{U}, x a^{L},-x \alpha,-x \beta\right)$

\section{Ranking Function}

A convenient method for comparing of the fuzzy numbers is by use of ranking functions. A ranking function is a map from $F(R)$ into the real line. The orders on $F(R)$ are:

$\tilde{a} \geq \tilde{b}$ if and only if $\mathfrak{R}(\tilde{a}) \geq \mathfrak{R}(\tilde{b})$

$\tilde{a}>\tilde{b}$ if and only if $\mathfrak{R}(\tilde{a})>\Re(\tilde{b})$

$\tilde{a}=\tilde{b}$ if and only if $\mathfrak{R}(\tilde{a})=\mathfrak{R}(\tilde{b})$

Where, $\tilde{a}$ and $\tilde{b}$ are in $F(R)$. It is obvious that $\tilde{a} \leq \tilde{b}$ if and only if $\tilde{b} \geq \tilde{a}$. Since there are many ranking function for comparing fuzzy numbers but robust ranking function is applied. Robust's ranking 
technique satisfies compensation, linearity and additive properties and provides results which are consistent with human intuition. Give a convex fuzzy number $\tilde{a}$, the Robust's Ranking index is defined by

$\Re(\tilde{a})=\int_{0}^{1} 0.5\left(a_{\alpha}^{L}, a_{\alpha}^{U}\right) d \alpha$, where $\left(a^{L}, a^{U}\right)$ is the $\alpha$ - level cut of the fuzzy number $\tilde{a}$.

In this paper this method for ranking the objective values. The Robust's ranking index $\mathfrak{R}(\tilde{a})$ gives the representative value of the fuzzy number $\tilde{a}$. It satisfies the linearity and additive property.

\section{Fuzzy Linear Programming Problems}

However, when formulating a mathematical programming problem which closely describes and represents a real-world decision situation, various factors of the real world system should be reflected in the description of objective functions and constraints involve many parameters whose possible values may assigned by experts. In the conventional approaches, such parameters are required to be fixed at some values in an experimental and subjective manner through the experts' understanding of the nature of the parameters in the problem-formulation process.

It must be observed that, in most real-world situations, the possible values of these parameters are often only imprecisely known to the experts. With this observation in mind, it would be certainly more appropriate to interpret the experts' understanding of the parameters as fuzzy numerical data which can be represented by means of fuzzy sets of the real line known as fuzzy numbers.

\section{Definition 4.1 Linear programming}

A linear programming (LP) problem is defined as:

$\operatorname{Max} z=c x$

s.t. $A x=b$

$x \geq 0$

Where, $c=\left(c_{1}, c_{2}, \ldots \ldots c_{n}\right), b=\left(b_{1}, b_{2}, \ldots \ldots b_{m}\right)^{T}$, and $A=\left[a_{i j}\right]_{m \times n}$.

In the above problem, all of the parameters are crisp. Now, if some of the parameters be fuzzy numbers then fuzzy linear programming is obtained which is defined in the next section.

\section{Definition 4.2 Fuzzy linear programming}

Suppose that in the linear programming problem some parameters be fuzzy numbers. Hence, it is possible that some coefficients of the problem in the objective function, technical coefficients the right hand side coefficients or decision making variables be fuzzy number [7], [8], [12] and [14]. Here, the linear programming problems with fuzzy numbers in the objective function.

Definition 4.3 Fuzzy number linear programming

A fuzzy number linear programming (FNLP) problem is defined as follows:

$\operatorname{Max} \tilde{z}=\tilde{c} x$

s.t. $A x=b$

$x \geq 0$

where, $b \in R^{m}, x \in R^{n}, A \epsilon R^{m \times n}, \tilde{c}^{T} \epsilon\left((F(R))^{n}\right.$, and $\Re$ is a Robust ranking function.

Definition 4.4 Fuzzy feasible solution

The vector $x \in R^{n}$ is a feasible solution to FNLP if and only if $x$ satisfies the constraints of the problem.

Definition 4.5 Fuzzy optimal solution

A feasible solution $x^{*}$ is an optimal solution for FNLP, if for all feasible solution $x$ for FNLP, then $\tilde{c} x^{*} \geq \tilde{c} x$.

Definition 4.6 Fuzzy basic feasible solution 
The basic feasible solution for FNLP problems is defined as:

Consider the system $A x=b$ and $x \geq 0$, where $A$ is an $m \times n$ matrix and $b$ is an $m$ vector. Now, suppose that $\operatorname{rank}(A, b)=\operatorname{rank}(A)=m$. Partition after possibly rearranging the columns of $A$ as $[B, N]$ where $B, m \times m$, is nonsingular. It is obvious that $\operatorname{rank}(B)=m$. The point $x=\left(x_{B}^{T}, x_{N}^{T}\right)^{T}$ where, $x_{B}=B^{-1} b$, $x_{N}=0$ is called a basic solution of the system. If $x_{B} \geq 0$, then $x$ is called a basic feasible solution (BFS) of the system. Here $B$ is called the basic matrix and $N$ is called the non basic matrix.

\section{Numerical Problem}

\section{Consider the FNLP}

$\operatorname{Max} \tilde{z}=(3,5,6,7) \tilde{x}_{1}+(5,8,11,12) \tilde{x}_{2}$

Subject to the constraints

$-2 \tilde{x}_{1}+3 \tilde{x}_{2} \leq 6$

$5 \tilde{x}_{1}+4 \tilde{x}_{2} \leq 10$

$\forall \tilde{x}_{1}, \tilde{x}_{2} \geq 0$.

Solution The optimal solution of the given fuzzy linear programming problem is evaluated in the Table 2 .

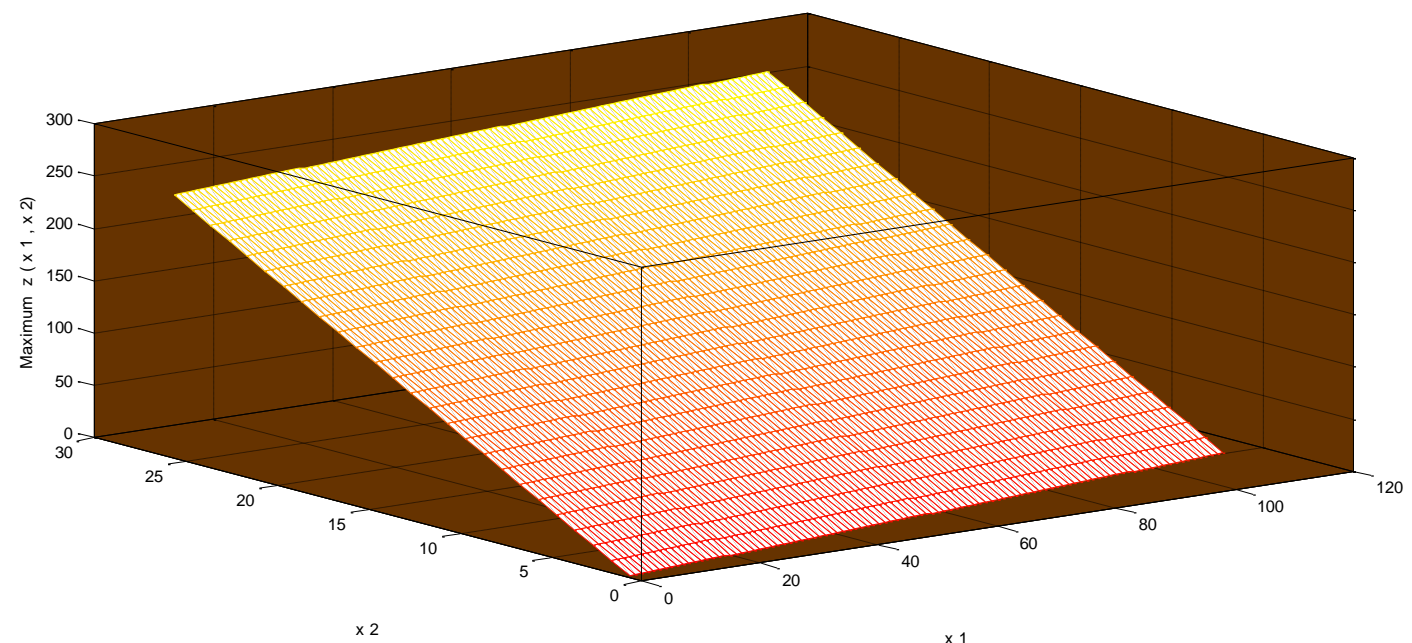

Figure 1 Mesh Plot of Maximum Fuzzy Total Profit, $\tilde{x}_{1}$ and $\tilde{x}_{2}$.

Table 2 Optimal Values for the Proposed Fuzzy Linear Programming Model

\begin{tabular}{|c|c|c|c|c|c|c|c|}
\hline \multicolumn{3}{|c|}{$\widetilde{\boldsymbol{C}}_{j}$} & $(3,5,6,7)$ & $(5,8,11,12)$ & $(0,0,0,0)$ & $(0,0,0,0)$ & \\
\hline$\widetilde{\boldsymbol{C}}_{B}$ & $\widetilde{\boldsymbol{Y}}_{B}$ & $\widetilde{\boldsymbol{X}}_{B}$ & $\widetilde{y}_{1}$ & $\widetilde{y}_{2}$ & $\widetilde{\boldsymbol{y}}_{3}$ & $\widetilde{\boldsymbol{y}}_{4}$ & $\begin{array}{l}\text { Min } \\
\text { ratio }\end{array}$ \\
\hline$(0,0,0,0)$ & $\tilde{y}_{3}$ & 6 & -2 & 3 & 1 & 0 & $2 \rightarrow$ \\
\hline$(0,0,0,0)$ & $\tilde{y}_{4}$ & 10 & 5 & 4 & 0 & 1 & 2.5 \\
\hline \multicolumn{2}{|l|}{$\tilde{z}_{j}$} & $(0,0,0,0)$ & $(-7,-6,-5,-3)$ & $\begin{array}{c}(-12,-11,-8,-5) \\
\uparrow\end{array}$ & $(0,0,0,0)$ & $(0,0,0,0)$ & $\tilde{\Delta}_{j}$ \\
\hline$\widetilde{\boldsymbol{C}}_{B}$ & $\widetilde{\boldsymbol{Y}}_{\boldsymbol{B}}$ & $\widetilde{\boldsymbol{X}}_{B}$ & $\widetilde{y}_{1}$ & $\tilde{\boldsymbol{y}}_{2}$ & $\widetilde{\boldsymbol{y}}_{3}$ & $\widetilde{y}_{4}$ & $\begin{array}{l}\text { Min } \\
\text { ratio }\end{array}$ \\
\hline$(5,8,11,12)$ & $\tilde{y}_{2}$ & 2 & $-\frac{2}{3}$ & 1 & $\frac{1}{3}$ & 0 & - \\
\hline$(0,0,0,0)$ & $\tilde{y}_{4}$ & 2 & \begin{tabular}{|l|}
$\frac{23}{3}$ \\
\end{tabular} & 0 & $-\frac{4}{3}$ & 1 & $\begin{array}{c}0.26 \\
\rightarrow\end{array}$ \\
\hline \multicolumn{2}{|l|}{$\tilde{z}_{j}$} & $(10,16,22,24)$ & $\left(-15, \frac{-40}{3}, \frac{-31}{3}, \frac{-19}{3}\right) \uparrow$ & $(0,0,0,0)$ & $\left(\frac{5}{3}, \frac{8}{3}, \frac{11}{3}, \frac{12}{3}\right)$ & $(0,0,0,0)$ & $\tilde{\Delta}_{j}$ \\
\hline$\widetilde{\boldsymbol{C}}_{B}$ & $\widetilde{\boldsymbol{Y}}_{B}$ & $\widetilde{\boldsymbol{X}}_{B}$ & $\tilde{\boldsymbol{y}}_{1}$ & $\tilde{y}_{2}$ & $\tilde{\boldsymbol{y}}_{3}$ & $\widetilde{\boldsymbol{y}}_{4}$ & $\begin{array}{l}\text { Min } \\
\text { ratio }\end{array}$ \\
\hline$(5,8,11,12)$ & $\tilde{y}_{2}$ & $\frac{50}{23}$ & 0 & 1 & $\frac{5}{23}$ & $\frac{2}{23}$ & - \\
\hline$(3,5,6,7)$ & $\tilde{y}_{1}$ & $\frac{6}{23}$ & 1 & 0 & $\frac{-4}{23}$ & $\frac{3}{23}$ & - \\
\hline
\end{tabular}




\begin{tabular}{|l|l|l|l|l|l|l|}
\hline$\tilde{z}_{j}$ & $\left(\frac{268}{23}, \frac{430}{23}, \frac{586}{23}, \frac{642}{23}\right)$ & $(0,0,0,0)$ & $(0,0,0,0)$ & $\left(\frac{-3}{23}, \frac{16}{23}, \frac{35}{23}, \frac{48}{23}\right)$ & $\left(\frac{19}{23}, \frac{31}{23}, \frac{40}{23}, \frac{66}{23}\right)$ & $\tilde{\Delta}_{j}$ \\
\hline
\end{tabular}

Therefore, the fuzzy optimal solution is $\tilde{x}_{1}^{*}=\frac{6}{23} \approx 0.2609, \quad \tilde{x}_{2}^{*}=\frac{50}{23} \approx 2.1739$ and $\tilde{z}^{*}=$ $\left(\frac{268}{23}, \frac{430}{23}, \frac{586}{23}, \frac{642}{23}\right)=\frac{963}{46} \approx 20.9348$.

\section{Sensitivity Analyses}

The investigations that deal with changes in the optimum solutions due to discrete variations in the parameter $b_{i}$ and the fuzzy parameter $\tilde{c}_{j}$ is called post optimal.

\subsection{Discrete Variation in $\tilde{\boldsymbol{c}}$}

The investigations that deal with changes in the optimum solutions due to discrete variations in the fuzzy parameter $\tilde{c}_{j}$ is called post optimal analysis.

Consider the fuzzy linear programming problem Maximize $\tilde{z}=\widetilde{\boldsymbol{C}}^{T} \widetilde{\boldsymbol{X}}_{\text {subject }}$ to $\boldsymbol{A} \widetilde{\boldsymbol{X}}=\boldsymbol{b}$ and $\widetilde{\boldsymbol{X}} \geq$ 0. There are two possibilities: i) $\tilde{c}_{1} \notin \tilde{\boldsymbol{c}}_{\boldsymbol{B}}$ and, ii) $\tilde{c}_{1} \in \tilde{\boldsymbol{c}}_{\boldsymbol{B}}$.

i) $\tilde{c}_{k} \notin \tilde{\boldsymbol{c}}_{\boldsymbol{B}}$, then the current solutions remain optimum for the new problem if $\tilde{z}_{k}-\left(\tilde{c}_{k}+\widetilde{\Delta c_{k}}\right) \geq$ 0 . Further, since $\tilde{z}$ is independent of $\tilde{c}_{k}$, the value of the objective function and the fuzzy optimum solution will be remain unchanged.

ii) $\tilde{c}_{k} \in \tilde{\boldsymbol{c}}_{\boldsymbol{B}}$, the current basic feasible solutions remain optimum for the new problem, $\tilde{\Delta}_{j} \geq 0$. That is $\operatorname{Max}\left\{\frac{-\tilde{\Delta}_{j}}{\tilde{y}_{k j}>0}\right\} \leq \Delta \tilde{c}_{k} \leq \operatorname{Min}\left\{\frac{-\tilde{\Delta}_{j}}{\tilde{y}_{k j}<0}\right\}$.

From the Table 2 it is observed that:

Variation in $\tilde{\boldsymbol{c}}_{\mathbf{1}}$ : since $\tilde{c}_{1} \in \tilde{\boldsymbol{c}}_{\boldsymbol{B}}$, the range of ${\widetilde{\Delta c_{1}}}_{1}$ is given by

$\operatorname{Max}_{y_{1 j}>0}\left\{\frac{-\tilde{\Delta}_{j}}{y_{1 j}}\right\} \leq \widetilde{\Delta c}_{1} \leq \operatorname{Min}_{y_{1 j}<0}\left\{\frac{-\tilde{\Delta}_{j}}{y_{1 j}}\right\}=\operatorname{Max}\left[\frac{-39 / 23}{3 / 23}\right] \leq \widetilde{\Delta c}_{1} \leq \operatorname{Min}\left[\frac{-24 / 23}{-4 / 23}\right]=-13 \leq \widetilde{\Delta c}_{1} \leq 6=-13 \leq$ $\widetilde{\Delta c_{1}} \leq 6$.

Variation in $\tilde{\boldsymbol{c}}_{2}$ : since $\tilde{c}_{2} \in \tilde{\boldsymbol{c}}_{\boldsymbol{B}}$, the range of $\widetilde{\Delta c_{2}}$ is given by

$\left.\left.\operatorname{Max}_{y_{2 j}>0}\left\{\frac{-\tilde{\Delta}_{j}}{y_{2 j}}\right\} \leq \widetilde{\Delta c}_{2} \leq \operatorname{Min}_{y_{2 j}<0}\left\{\frac{-\tilde{\Delta}_{j}}{y_{2 j}}\right\}=\operatorname{Max}\left[\frac{-\frac{24}{23}}{\frac{5}{23}}, \frac{-\frac{39}{23}}{\frac{2}{23}}\right] \leq \widetilde{\Delta c}_{2} \leq \infty\right]=\operatorname{Max}\left[\frac{-24}{5}, \frac{-39}{2}\right] \leq \widetilde{\Delta c}_{2} \leq \infty\right]=$ $-\frac{24}{5} \leq \widetilde{\Delta c}_{2} \leq \infty=-4.8 \leq \widetilde{\Delta c}_{2} \leq \infty$.

Hence, $-13 \leq \widetilde{\Delta c_{1}} \leq 6$ and $-4.8 \leq \widetilde{\Delta c_{2}} \leq \infty$. Now, since $\tilde{c}_{1}=(3,5,6,7)$ and $\tilde{c}_{2}=(5,8,11,12)$ the required range of variation is $-\frac{31}{4} \leq \tilde{c}_{1} \leq \frac{45}{4}$ and $\frac{89}{20} \leq \tilde{c}_{2} \leq \infty$. Figure 1 represents the mesh plot of three dimensional figure of fuzzy total profit, $x_{1}$ and $x_{2}$ respectively.

\section{Conclusions}

This paper has been implemented Robust's ranking technique for a linear programming problem with fuzzy parameters, which allows taking a decision interactively with the decision maker in fuzzy decision space. The decision maker also has additional information about the availability violation of the per unit profit factor in the multi-objective function, and about the compatibility of the cost of the solution with his wishes for the values of the objective function. The decision maker can arbitrate in all the steps of the decision process which makes our approach very useful to be applied in a lot of real-world problems where the information is uncertain with nonrandom, like environmental management, project management, marketing, production etc.. Finally, for all decisions about linear programming model and sensitivity analyses, it is important to adopt a framework rooted in a fuzzy decision space. Contrary to what many believe, market uncertainty is a manageable risk.

\section{References}

[1] M.S. Bazaraa, J.J. Jarvis, H.D. Sherali, "Linear Programming and Network Flows". John Weily, Second Edition, New York, 1990. 
[2] R.E. Bellman, L.A. Zadeh, "Decision making in a fuzzy environment". Management Science, (1970) 17: 141-164.

[3] A.N. Gani, C. Duraisamy, C. Veeramani, "A note on fuzzy linear programming problem using LR fuzzy number”. International Journal of Algorithms, Computing and Mathematics, (2009) 2 (3): 93-106.

[4] M. Jimenez, M. Arenas, A. Bilbao, M.V. Rodriguez, "Linear programming with fuzzy parameters: An interactive method resolution". European Journal of Operational Research, 2005.

[5] Y.J. Lai, C.L. Hwang, "A new approach to some possibilistic linear programming problem". Fuzzy Sets and Systems, (1992) 49.

[6] Y.J. Lai, C.L. Hwang, "Mathematical Programming Methods and Applications". Springer, Berlin, 1992.

[7] H.R. Maleki, "Ranking functions and their applications to fuzzy linear programming". Far. East Journal of Mathematical Science, (2002) 4: 283-301.

[8] H.R. Maleki, M. Tata, M. Mashinchi, "Linear programming with fuzzy variables". Fuzzy Sets and Systems, (2000) 109: 21-31.

[9] S.H. Nasseri, E. Ardil, A. Yazdani, R. Zaefarian, "Simplex method for solving linear programming problems with fuzzy numbers". World Academy of Science, Engineering and Technology, (2005) 10: 284-288.

[10] S.H. Nasseri, F. Taleshian, Z. Alizadeh, J. Vahidi, "A new method for ordering fuzzy number". The Journal of Mathematics and Computer Science, (2012) 4, (3): 283-294.

[11] M. Pattnaik, "Models of Inventory Control”. LAP Lambart Academic, Germany, 2012.

[12] R. Rangarajan, A. Solairaju, "Computing improved fuzzy optimal Hungarian assignment problems with fuzzy costs under robust ranking techniques". International Journal of Computer Applications, (2010) 6(4): 6-13.

[13] H. Rommelfanger, R. Hanuscheck, J. Wolf, "Linear programming with fuzzy objective". Fuzzy Sets and Systems, (1989) 29: 31-48.

[14] K. Swarup, P.K. Gupta, M. Mohan, “Operations Research”.Sultan Chand and Sons, New Delhi, 2006.

[15] J.L. Verdegay, "A dual approach to solve the fuzzy linear programming problem". Fuzzy Sets and Systems, (1984) 14: 131-141.

[16] E.R. Yanehsari, M.A. Lahirimi, "Union and intersection fuzzy subhypergroups". The Journal of Mathematics and Computer Science, (2012) 5, (2): 82-90.

[17] H.J. Zimmermann, "Fuzzy programming and linear programming with several objective functions". Fuzzy Sets and Systems, (1978) 1: 45-55. 